INPLASY

PROTOCOL

To cite: Zhu et al. The effectiveness and safety of Sanfu acupoint herbal patching for treating allergic rhinitis: a protocol for systematic review and MetaAnalysis. Inplasy protocol 2020100101. doi: 10.37766/inplasy2020.10.0101

Received: 26 October 2020

Published: 26 October 2020

Corresponding author: Qiaochu Zhu

735726477@qq.com

Author Affiliation: Hubei University Of Chinese Medicine

Support: Available.

Review Stage at time of this submission: The review has not yet started.

Conflicts of interest: None.

\section{The effectiveness and safety of Sanfu acupoint herbal patching for treating allergic rhinitis: a protocol for systematic review and Meta-Analysis}

Zhu, Q1; Song, A2; Zhou, Z3; Jiao, Y4; Wei, D5; Zhang, Y6; Wang, $Y^{7}$; Tian, $\mathrm{X}^{8}$.

Review question / Objective: To assess the effectiveness and safety of sanfu acupoint herbal patching for treating allergic rhinitis.

Condition being studied: Allergic rhinitis (AR) is an inflammatory disease of the nasal mucosa which main symptoms are nasal congestion, runny nose, nasal itching, and sneezing. According to epidemiological surveys, $10 \%-25 \%$ of patients suffer from this disease.Western medicine treatment is mainly based on hormone therapy and immunodesensitization therapy, but its adverse effects to the human body are unpredictable. Nowadays studies show that Sanfu acupoint herbal patching has obvious advantages in the prevention and treatment of allergic rhinitis.

INPLASY registration number: This protocol was registered with the International Platform of Registered Systematic Review and Meta-Analysis Protocols (INPLASY) on 26 October 2020 and was last updated on 26 October 2020 (registration number INPLASY2020100101).

\section{INTRODUCTION}

Review question / Objective: To assess the effectiveness and safety of sanfu acupoint herbal patching for treating allergic rhinitis.
Condition being studied: Allergic rhinitis (AR) is an inflammatory disease of the nasal mucosa which main symptoms are nasal congestion, runny nose, nasal itching, and sneezing. According to epidemiological surveys, $10 \%-25 \%$ of patients suffer from this disease.Western 
medicine treatment is mainly based on hormone therapy and immunodesensitization therapy, but its adverse effects to the human body are unpredictable. Nowadays studies show that Sanfu acupoint herbal patching has obvious advantages in the prevention and treatment of allergic rhinitis.

\section{METHODS}

Search strategy: We will search the following databases from January 2010 to October 2020: Pubmed, Web of Science, Cochrane Library, EMBASE, Medline, China National Knowledge Infrastructure (CNKI), VIP Database, WANFANG Database, China Biology Medicine disc(CBM). And clinical researches which are not completed will be searched on the mainstream registries such as Chinese Clinical Trail Registry (http://www.chictr.org.cn/searchproj.aspx) and ClinicalTrials.gov trials registry (http:// www.clinicaltrials.gov).

Participant or population: Patients diagnosed with allergic rhinitis regardless of nation, gender, race, courses of disease, as long as they are in accordance with the diagnostic criteria for allergic rhinitis can be included into the study.

Intervention: Sanfu acupoint herbal patching(No restriction on herbal medicine composition, course of disease, selection of acupoints, etc.) or Sanfu herbal patching plus other same therapy as control group (such as oral Chinese medicine, moxibustion, acupuncture, placebo, western medicine).

Comparator: Common therapy(oral Chinese medicine, moxibustion, acupuncture, sham acupoint herbal patching, western medicine, cupping, etc.) or no treatment Sanfu acupoint herbal application plus common therapy as experimental group, meanwhile the same common therapy in the control group involved.

Study designs to be included: All of the randomized controlled trails(RCTs) that comply with the inclusion criteria will be included into this research.

Eligibility criteria: Only RCTs complied with the following requirements will be eligible for the research: Participants who suffered from allergic rhinitis, Sanfu acupoint herbal patching or Sanfu acupoint herbal patching plus other common therapy(such as oral Chinese medicine, moxibustion, acupuncture, placebo, western medicine) are treated as experimental group while placebo plus other same common therapy or no treatment are employed as comparisons will be included.

Information sources: The following 9 electronic databases will be searched: Pubmed, Web of Science, Cochrane Library, EMBASE, Medline, China National Knowledge Infrastructure (CNKI), VIP Database, WANFANG Database, China Biology Medicine disc(CBM), and the ongoing clinical researches will be searched on the mainstream registries such as Chinese Clinical Trail Registry (http://www.chictr.org.cn/searchproj.aspx) and ClinicalTrials.gov trials registry (http:// www.clinicaltrials.gov). We will contact with first author if we cannot find full text after comprehensive searching. No grey literature will be included.

Main outcome(s): Total effective rate.

Additional outcome(s): (1). TNSS(Total Nasal Symptom Score); (2). Recurrence rate; (3). RQLQ(Rhinitis Quality of Life Questionnaire); (4).Laboratory indicators: IgE; TNF-a, IFN-, IL-4, IL-5, etc.; (5). adverse events.

Data management: Two reviewers will extract data of included studies independently, and consensus will be reached by discussion with a third party if there is a dispute.

Quality assessment / Risk of bias analysis: We will use Cochrane risk-of-bias tool (ROB2.0) to evaluate the quality of included trails. 5 types of bias will appear in the randomization process: The bias caused by 
the deviation of intervention measures, the bias caused by the lack of result data, the bias caused by the measurement of the results, the bias caused by the selective reporting of the results. And a final summary of risk bias can be determined. Two reviewers will evaluate all included studies and consensus will be reached by discussion with third reviewer in case of controversy.

Strategy of data synthesis: Statistical analyses will be operated by STATA14.0 software. Dichotomous data will be expressed by the risk ratio (RR) and 95\% confidence interval (CI); Continuous data will be expressed by the mean difference (MD) of $95 \% \mathrm{Cl}$. If different measurement scales are used, standardized mean difference (SMD) will be operated to analyze it. I-squared test is used to evaluate the heterogeneity of included studies. If there is no significant heterogeneity $(12<50 \%)$, the fixed effects model is used. If there is a large heterogeneity $(12 \geq 50 \%)$, use a random effects model.

Subgroup analysis: If data is reasonable, we are going to conduct subgroup analysis of different types of groups, the following subgroups we will conduct: (1). Different treating time (2). Herbal medicine on patch (3). Different control measures( western medicine, placebo herbal patch, no treatment, acupunture, moxibustion, etc.)

Sensibility analysis: Sensitivity analysis can find the source of heterogeneity and confounding factors in the research. If data is substantial, the Stata14.0 software will be used for sensitivity analysis. If the heterogeneity is too large to find its source, we will consider giving up meta-analysis and adopting descriptive analysis.

Language: Chinese and English.

Country(ies) involved: China.

Keywords: Sanfu herbal patching; sanfu herbal acupoint application; allergic rhinitis; meta-analysis.
Contributions of each author:

Author 1 - Qiaochu Zhu.

Author 2 - Aiqun Song.

Author 3 - Zhongyu Zhou.

Author 4 - Yang Jiao.

Author 5 - Dan Wei.

Author 6 - Yangpu Zhang.

Author 7 - Yan Wang.

Author 8 - Xiaohui Tian. 